\title{
DERIVATION OF NEW QUANTUM HYDRODYNAMIC EQUATIONS USING ENTROPY MINIMIZATION*
}

\author{
ANSGAR JÜNGEL ${ }^{\dagger}$, DANIEL MATTHES ${ }^{\dagger}$, AND JOSIPA PINA MILIŠIĆ ${ }^{\dagger}$
}

\begin{abstract}
New quantum hydrodynamic equations are derived from a Wigner-Boltzmann model, using the quantum entropy minimization method recently developed by Degond and Ringhofer. The model consists of conservation equations for the carrier, momentum, and energy densities. The derivation is based on a careful expansion of the quantum Maxwellian in powers of the Planck constant. In difference to the standard quantum hydrodynamic equations derived by Gardner, the new model includes vorticity terms and a dispersive term for the velocity. Numerical current-voltage characteristics of a one-dimensional resonant tunneling diode for both the new quantum hydrodynamic equations and Gardner's model are presented. The numerical results indicate that the dispersive velocity term regularizes the solution of the system.
\end{abstract}

Key words. Quantum moment hydrodynamics, entropy minimization, quantum Maxwellian, moment method, finite-difference discretization, numerical simulations, resonant tunneling diode, current-voltage characteristics.

AMS subject classifications. 35Q40, 65M06, $76 \mathrm{Y} 05$.

1. Introduction. Quantum phenomena in semiconductor devices are increasingly important as the characteristic lengths of modern devices are of the order of deca-nanometers only. In fact, there are devices, like resonant tunneling diodes, whose behavior is essentially based on quantum effects. Since the numerical solution of the Schrödinger equation (or one of its approximations) or the Wigner equation is very time consuming, fluid-type quantum models seem to provide a compromise between accurate and efficient numerical simulations. Moreover, quantum fluid models have several advantages. First, they are formulated in macroscopic quantities like the current density, which can be measured. Second, for the macroscopic quantum models, the same type of boundary conditions are commonly employed as for their classical counterparts.

A fluiddynamical formulation of the Schrödinger equation is known since the early years of quantum mechanics [25]. A simple derivation uses WKB wave functions $\psi=\sqrt{n} \exp (i S / \varepsilon)$ for the electron density $n(x, t)$ and the phase $S(x, t)$, where $\varepsilon$ is the scaled Planck constant. Separating the real and the imaginary part of the single-state Schrödinger equation gives Euler-type equations for $n$ and the "velocity" $u=\nabla S$, which are called the quantum hydrodynamic (QHD) model. These equations include the so-called Bohm potential $\Delta \sqrt{n} / \sqrt{n}$ as a quantum correction $[16,19]$. In the semi-classical limit $\varepsilon \rightarrow 0$, the classical pressure-less Euler equations are recovered.

In order to incorporate many-particle effects, we are aware of two approaches. The first approach starts from the mixed-state Schrödinger-Poisson system [16, 19]. Defining the particle and current densities as the superpositions of all single-state densities, quantum equations for the macroscopic variables (particle density, current density, and energy density) are derived. The system of equations is closed by expressing the heat flux heuristically in terms of the macroscopic variables.

* The authors acknowledge partial support from the Deutsche Forschungsgemeinschaft (DFG), grants JU359/3 (Gerhard-Hess Award) and JU359/5 (Priority Program "Multi-Scale Problems") and from the Forschungsfond of the University of Mainz.

${ }^{\dagger}$ Institut für Mathematik, Universität Mainz, Staudingerweg 9, 55099 Mainz, Germany; e-mail: \{juengel, matthes, milisic\}@mathematik. uni-mainz.de. 
The second approach starts from the (collisional) Wigner equation in positionmomentum space,

$$
\partial_{t} f+p \cdot \nabla_{x} f+\theta[V] f=Q(f), \quad t>0, \quad f(x, p, 0)=f_{I}(x, p), \quad(x, p) \in \mathbb{R}^{2 d},
$$

where $(x, p)$ is the position-momentum variable, $t>0$ is the time, and $\theta[V]$ is a pseudo-differential operator defined by

$$
\begin{aligned}
& (\theta[V] w)(x, p, t)= \\
& \frac{i}{(2 \pi)^{d / 2}} \int_{\mathbb{R}^{2 d}} \frac{1}{\varepsilon}\left[V\left(x+\frac{\varepsilon}{2} \eta, t\right)-V\left(x-\frac{\varepsilon}{2} \eta, t\right)\right] w\left(x, p^{\prime}, t\right) e^{i \eta \cdot\left(p-p^{\prime}\right)} d \eta d p^{\prime} .
\end{aligned}
$$

The electric potential $V=V(x, t)$ is selfconsistently coupled to the Wigner function $f(x, p, t)$ via Poisson's equation

$$
\lambda^{2} \Delta V=\int_{\mathbb{R}^{d}} f d p-C,
$$

where $\lambda$ is the scaled Debye length and $C=C(x)$ the doping concentration characterizing the semiconductor device. Notice that the collision-less Wigner equation is formally equivalent to the Heisenberg equation for the density matrix.

The above approach allows for an abstract formulation of the collision operator. In fact, we only assume that its kernel consists of the quantum thermal equilibrium distribution (defined in section 2) and that the operator preserves certain moments.

The macroscopic variables are defined as the moments of the Wigner function over momentum space; more precisely, we consider the particle density $n=\langle 1\rangle$, the fluiddynamical momentum density $n u=\langle p\rangle$, and the energy density $e=\left\langle\frac{1}{2}|p|^{2}\right\rangle$, where we have used the notation $\langle g(p)\rangle=\int f(\cdot, p) g(p) d p$ for functions $g(p)$. In order to obtain macroscopic equations as well, a moment method is applied to (1.1): we multiply the equation by $1, p$, and $\frac{1}{2}|p|^{2}$ and integrate over momentum space. This yields evolution equations for $n, n u$ and $e$. However, the resulting system of moment equations needs to be closed.

As a closure condition, Gardner [11] employed a quantum-corrected thermal equilibrium distribution function in place of $f$ in the derivation of the moment equations. The use of this closure can be - formally - justified by a hydrodynamic scaling and passage to the limit of vanishing scaling parameter. Gardner bases his choice of the quantum equilibrium distribution on a result by Wigner [30]. His equilibrium function contains second derivatives of the electric potential. However, the total potential is discontinuous at heterojunctions. Arguing that the electric potential is close to $\log n$ near equilibrium, he replaces $V$ by $\log n$, which is the origin of the Bohm potential.

Another approach, avoiding second derivatives of the potential, consists in deriving an approximate solution to the Bloch equation by an asymptotic expansion of the solution for "small" potentials. This leads to the so-called "smooth" QHD equations in which the potential $V$ is replaced by a smoothed potential $S[V]$, where $S$ is a pseudo-differential operator [13]. The drawback of this approach is that the numerical solution of the "smooth" QHD model is a non-trivial task. Moreover, there is an ambiguity in the interpretation of the temperature (see the remark in section 6 of $[27])$.

Our approach to define a closure is based on Levermore's entropy minimization principle. This method has been first employed in the context of classical gas dynamics [24] and has been recently extended to quantum fluids by Degond and Ringhofer 
[8]. The idea is to define the equilibrium distribution as the minimizer $M_{f}$ of the quantum entropy subject to the constraints of given moments. (Here, we adopted the mathematical sign convention of decreasing entropy.) The minimizer is called the quantum Maxwellian since there are some similarities to the classical Maxwellian of gas dynamics (see section 2). The quantum Maxwellian $M_{f}$, as the solution of a constrained minimization problem, depends on Lagrange multipliers which can be interpreted in the $O\left(\varepsilon^{2}\right)$ approximation as the logarithm of the particle density, the fluid velocity, and the temperature, respectively. Expanding $M_{f}$ in powers of $\varepsilon^{2}$ and assuming similarly as in [11] that spatial variations of the temperature $T=T(x, t)$ are of the order $O\left(\varepsilon^{2}\right)$, we derive the following QHD equations up to order $O\left(\varepsilon^{4}\right)$,

$$
\begin{aligned}
\partial_{t} n+\operatorname{div}(n u) & =0, \\
\partial_{t}(n u)+\operatorname{div}(n u \otimes u)+\operatorname{div} P-n \nabla V & =0, \\
\partial_{t} e+\operatorname{div}((P+e I) u)+\operatorname{div} S-\left(\frac{d}{2}+1\right) \operatorname{div} U-n u \cdot \nabla V & =0,
\end{aligned}
$$

where $I$ is the unit matrix in $\mathbb{R}^{d}$, the energy density equals

$$
e=\frac{d}{2} n T+\frac{1}{2} n|u|^{2}-\frac{\varepsilon^{2}}{24} n\left(\Delta \log n-\frac{1}{T} \operatorname{tr}\left(R^{\top} R\right)\right),
$$

with the trace "tr" of a matrix, the quantities $P$ (stress tensor), $S$, and $U$ are given by

$$
\begin{aligned}
P & =n T I-\frac{\varepsilon^{2}}{12} n\left((\nabla \otimes \nabla) \log n-\frac{1}{T} R^{\top} R\right), \\
S & =-\frac{\varepsilon^{2}}{12} n\left(\left(\frac{d}{2}+1\right) R \nabla \log n+\left(\frac{d}{2}+2\right) \operatorname{div} R+\frac{3}{2} \Delta u\right), \\
U & =-\frac{\varepsilon^{2}}{12} n(R \nabla \log n+\operatorname{div} R),
\end{aligned}
$$

and the vorticity matrix $R=\left(R_{i j}\right)$ is the antisymmetric part of the velocity derivative,

$$
R_{i j}=\partial_{x_{j}} u_{i}-\partial_{x_{i}} u_{j}
$$

A more general model, allowing arbitrarily large spatial deviations of the temperature, is derived in section 3 .

The quantum correction $\left(\varepsilon^{2} / 12\right) n(\nabla \otimes \nabla) \log n$ to the stress tensor in the QHD equations has been first stated in the semiconductor context by Ancona, Iafrate, and Tiersten in $[1,2]$. Since

$$
\frac{\varepsilon^{2}}{12} \operatorname{div}(n(\nabla \otimes \nabla) \log n)=\frac{\varepsilon^{2}}{6} n \nabla\left(\frac{\Delta \sqrt{n}}{\sqrt{n}}\right),
$$

the quantum correction can be interpreted as a force including the Bohm potential $\Delta \sqrt{n} / \sqrt{n}[10]$. The hydrodynamic formulation of quantum mechanics is employed in solid-state physics since many years; see, for instance, [17] and the references in the review [21].

For $\varepsilon=0$ in (1.3)-(1.5), we recover the classical hydrodynamic equations. For $\varepsilon>0$ and constant temperature, we obtain the same equations as derived in [20] where also the quantum entropy minimization method has been used. Our model 
differs from Gardner's QHD equations (formulas (1)-(3) in [11]) by the vorticity term $R$ and the dispersive velocity term in the energy equation (1.5),

$$
\operatorname{div} q_{S}=\frac{\varepsilon^{2}}{8} \operatorname{div}(n \Delta u)
$$

The origin of this difference lies in the different choices of the quantum Maxwellian. We refer to section 3.5 for a detailed discussion.

The term $q_{S}$ - but not the vorticity $R$ - also appears in other QHD derivations. It has been derived in [12] from a mixed-state Wigner model and interpreted as a dispersive "heat flux" (see formula (36) in [12]). Moreover, it appears in the QHD equations of [15] involving a "smoothed" potential, derived from the Wigner-Boltzmann equation by a Chapman-Enskog expansion.

An interesting feature of the dispersive term (1.7) is that it stabilizes the QHD system numerically. This statement needs some explaination. It is known that the numerical approximation of Gardner's QHD model (see (6.5)-(6.7)) is quite delicate. The usual approach is to employ a hyperbolic solver, for instance an upwind method [11] or a shock-capturing discontinuous Galerkin method [5], originally devised for the classical hydrodynamic equations. It has been argued in [23] that a hyperbolic solver may be inadequate for the QHD equations since the numerical viscosity might destroy the dispersive quantum effects. Therefore, a central finite-difference scheme provides an alternative (but still simple) numerical approach. In fact, a central finite-difference approach for Gardner's QHD equations fails and a stabilization in form of numerical viscosity seems to be necessary. The dispersive term (1.7) allows us to solve the new QHD equations by using a central scheme, thus avoiding numerical viscosity.

In this paper we present the first numerical simulations of a QHD model involving the term (1.7). More precisely, a simple one-dimensional resonant tunneling diode is simulated. The current-voltage characteristics show multiple regions of negative differential resistance. The dispersive term (1.7) has the effect of "smoothing" the current-voltage curve, i.e., it decreases the peak-to-valley ratio, the quotient of the peak to the valley current.

Another QHD model with physical viscosity has been derived in [18] using a Fokker-Planck collision operator. This operator describes the interaction of the electrons with a heat bath modeling the phonons of the semiconductor lattice. In numerical simulations of a resonant tunneling diode, it turns out that the shape of the current-voltage characteristic is unphysical if the temperature is kept constant [23], and that the diffusion effects are too strong compared to the quantum dispersion [22].

We also examine the existence of conserved quantities of the new QHD equations. Clearly, the mass is conserved. We prove that also the energy $E=\int\left(e+\lambda^{2}|\nabla V|^{2} / 2\right) d x$ is conserved. This provides gradient estimates for the particle density, velocity, and temperature, which is useful in the mathematical analysis of the equations.

We summarize the advantages of our approach:

- Starting from the Wigner-BGK equation, no ad-hoc assumptions are needed in order to derive the QHD equations.

- An energy for the new model can be defined, leading to useful mathematical estimates.

- The dispersive velocity term seems to stabilize the (numerical) solution of the system.

- The new model provides physically reasonable current-voltage characteristics.

The paper is organized as follows. In section 2 we specify our definition of the quantum Maxwellian which is used as the closure in the moment method developed in 
section 3. Section 4 is devoted to simplified QHD models and the system (1.3)-(1.5) is derived. In section 5 we prove that the energy of the system is conserved. Finally, in section 6, the new QHD model (1.3)-(1.5) is numerically discretized and solved in one space dimension, and simulations of a resonant tunneling diode are presented.

2. Definition of the quantum Maxwellian. In order to define the quantum Maxwellian, we first recall the Wigner transform. Let $A_{\rho}$ be an operator on $L^{2}\left(\mathbb{R}^{d}\right)$ with integral kernel $\rho\left(x, x^{\prime}\right)$, i.e.

$$
\left(A_{\rho} \phi\right)(x)=\int_{R^{d}} \rho\left(x, x^{\prime}\right) \phi\left(x^{\prime}\right) d x^{\prime} \quad \text { for all } \phi \in L^{2}\left(\mathbb{R}^{d}\right) .
$$

The Wigner transform of $A_{\rho}$ is defined by

$$
W\left(A_{\rho}\right)(x, p)=\frac{1}{(2 \pi)^{d}} \int_{\mathbb{R}^{d}} \rho\left(x+\frac{\varepsilon}{2} \eta, x-\frac{\varepsilon}{2} \eta\right) e^{i \eta \cdot p} d \eta .
$$

Its inverse $W^{-1}$, also called Weyl quantization, is defined as an operator on $L^{2}\left(\mathbb{R}^{d}\right)$ :

$$
\left(W^{-1}(f) \phi\right)(x)=\int_{\mathbb{R}^{2 d}} f\left(\frac{x+y}{2}\right) \phi(y) e^{i p \cdot(x-y) / \varepsilon} d p d y \quad \text { for all } \phi \in L^{2}\left(\mathbb{R}^{d}\right) .
$$

With these definitions we are able to introduce the quantum exponential and the quantum logarithm formally by

$$
\operatorname{Exp} f=W\left(\exp W^{-1}(f)\right), \quad \log f=W\left(\log W^{-1}(f)\right),
$$

where exp and log are the operator exponential and logarithm, respectively. In [7] it has been (formally) shown that the quantum exponential and quantum logarithm are equal to the usual exponential and logarithm, respectively, up to order $O\left(\varepsilon^{2}\right)$,

$$
\operatorname{Exp} f=\exp f+O\left(\varepsilon^{2}\right), \quad \log f=\log f+O\left(\varepsilon^{2}\right) .
$$

The essential ingredient in the definition of the quantum Maxwellian is the relative quantum entropy. Let a quantum mechanical state be described by the Wigner function $f$ solving the Wigner equation (1.1). Then its relative quantum (von Neumann) entropy is given by

$$
H(f)=\int_{\mathbb{R}^{2 d}} f(x, p)\left((\log f)(x, p)-1+\frac{|p|^{2}}{2}-V(x)\right) d x d p .
$$

Whereas the classical entropy is a function on the configuration space, the above quantum entropy is a real number, underlining the non-local nature of quantum mechanics.

We define the quantum thermal equilibrium or quantum Maxwellian $M_{f}$ for some given function $f(x, p)$ as the solution of the constrained minimization problem

$$
H\left(M_{f}\right)=\min \left\{H(\hat{f}): \int_{\mathbb{R}^{d}} \hat{f}(x, p, t)\left(\begin{array}{c}
1 \\
p \\
|p|^{2} / 2
\end{array}\right) d p=\left(\begin{array}{c}
n(x, t) \\
n u(x, t) \\
e(x, t)
\end{array}\right), x \in \mathbb{R}^{d}, t>0\right\},
$$


where

$$
\begin{aligned}
n(x, t) & =\langle 1\rangle(x, t)=\int_{\mathbb{R}^{d}} f(x, p, t) d p, \\
n u(x, t) & =\langle p\rangle(x, t)=\int_{\mathbb{R}^{d}} f(x, p, t) p d p, \\
e(x, t) & =\frac{1}{2}\left\langle|p|^{2}\right\rangle(x, t)=\frac{1}{2} \int_{\mathbb{R}^{d}} f(x, p, t)|p|^{2} d p .
\end{aligned}
$$

In $[8]$ it is shown that the solution $f^{*}$ of the contrained minimization problem (if it exists) is given by

$$
M_{f}(x, p, t)=\operatorname{Exp}\left(A(x, t)-\frac{|p-w(x, t)|^{2}}{2 T(x, t)}\right) .
$$

The Lagrange multipliers $A, w$, and $T$ are uniquely determined by the moments of $f$. They can be interpreted (up to order $O\left(\varepsilon^{2}\right)$ ) as the logarithm of the particle density, the velocity, and the temperature, respectively (see Lemma 3.4).

3. Derivation of the general QHD model. The derivation of the new QHD equations is done in several steps. First, we derive the moment equations. Then the quantum exponential is expanded in powers of $\varepsilon^{2}$ up to order $O\left(\varepsilon^{4}\right)$. The third step is to expand the moments accordingly. Finally, the expansions are substituted into the moment equations.

3.1. Moment equations. We consider the Wigner-Boltzmann equation (1.1) in the hydrodynamic scaling, i.e., we introduce the scaling

$$
x^{\prime}=\delta x, \quad t^{\prime}=\delta t,
$$

for some parameter $\delta>0$ which is assumed to be small compared to one. Then (1.1) becomes for $f=f_{\delta}$ (omitting the primes)

$$
\partial_{t} f_{\delta}+p \cdot \nabla_{x} f_{\delta}+\theta[V] f_{\delta}=\delta^{-1} Q\left(f_{\delta}\right), \quad(x, p) \in \mathbb{R}^{2 d}, t>0,
$$

with initial condition $f_{\delta}(x, p, 0)=f_{I}(x, p)$. We assume that the collision operator has the following properties: Its kernel consists exactly of (multiples of) $M_{f}$ and

$$
\int_{\mathbb{R}^{d}} Q(f) d p=0, \quad \int_{\mathbb{R}^{d}} Q(f) p d p=0, \quad \int_{\mathbb{R}^{d}} Q(f) \frac{1}{2}|p|^{2} d p=0 \quad \text { for all } f(x, p) .
$$

An example satisfying these conditions is the relaxation-time or "BGK" operator $Q(f)=M_{f}-f$ (with scaled relaxation time $\tau=1$ ) [4]. It is possible to relax the conditions (3.2) by assuming that the above integrals can be expressed in terms of $n$, $n u$, and $e$. For instance, in [15] it is assumed that the first moments of the collision operator yield the relaxation-time terms

$$
\int_{\mathbb{R}^{d}} Q(f) d p=0, \quad \int_{\mathbb{R}^{d}} Q(f) p d p=-\frac{n u}{\tau_{p}}, \quad \int_{\mathbb{R}^{d}} Q(f) \frac{1}{2}|p|^{2} d p=-\frac{1}{\tau_{w}}\left(e-\frac{3}{2} n T_{0}\right),
$$

where $\tau_{p}$ and $\tau_{w}$ are the momentum and energy relaxation times, respectively, and $T_{0}$ is the lattice temperature.

The formal limit $\delta \rightarrow 0$ in (3.1) yields $Q(f)=0$, where $f=\lim _{\delta \rightarrow 0} f_{\delta}$, which implies that the limit $f$ is equal to $M_{f}$. 
The moment equations are obtained from (3.1) by multiplication with $1, p$, and $\frac{1}{2}|p|^{2}$, respectively, and integration over the momentum space. Since

$$
\int_{\mathbb{R}^{d}} \theta[V] f d p=0, \quad \int_{\mathbb{R}^{d}} \theta[V] f p d p=-n \nabla V, \quad \int_{\mathbb{R}^{d}} \theta[V] f \frac{1}{2}|p|^{2} d p=-n u \cdot \nabla V
$$

(see, e.g., [7]), we obtain

$$
\begin{aligned}
\partial_{t} n+\operatorname{div}(n u) & =0, \\
\partial_{t}(n u)+\operatorname{div}\langle p \otimes p\rangle-n \nabla V & =0, \\
\partial_{t} e+\operatorname{div}\left\langle\frac{1}{2}|p|^{2} p\right\rangle-n u \cdot \nabla V & =0,
\end{aligned}
$$

where $(p \otimes p)_{i j}=p_{i} p_{j}$ for $i, j=1, \ldots, d$. Recall that the brackets denote integration against the Wigner function $f=M_{f}$, i.e. in multi-index notation,

$$
\left\langle p^{\alpha}\right\rangle(x, t)=\int_{\mathbb{R}^{d}} M_{f}(x, p, t) p^{\alpha} d p
$$

for multi-indices $\alpha \in \mathbb{N}^{d}$. To close the system (3.3)-(3.5), we need to express the integrals $\langle p \otimes p\rangle$ and $\left\langle\frac{1}{2}|p|^{2} p\right\rangle$ in terms of the moments $n, n u$, and $e$. This constitutes the main step of the derivation.

The following computations are simplified by working with the new variable $s=$ $T^{-1 / 2}(p-w)$, where $w$ is the Lagrange multiplier introduced in (2.3). In terms of $s$, the quantum Maxwellian reads as

$$
M_{f}(x, p(s))=\operatorname{Exp}\left(A(x)-\frac{1}{2}|s|^{2}\right)=: g(x, s) .
$$

From now on, we omit the dependence of the time $t$ since it acts only as a parameter. The substitution $p \mapsto s$ yields

$$
\left\langle s^{\alpha}\right\rangle(x)=T^{d / 2} \int_{\mathbb{R}^{d}} g(x, s) s^{\alpha} d s .
$$

In the following lemma we express the moments $\left\langle p^{\alpha}\right\rangle$ in terms of moments in $s$. This allows for a more canonical form of the QHD equations.

LEMMA 3.1. The system (3.3)-(3.5) has the formal expansion

$$
\begin{aligned}
\partial_{t} n+\operatorname{div}(n u) & =0, \\
\partial_{t}(n u)+\operatorname{div}(n u \otimes u)+\operatorname{div} P-n \nabla V & =O\left(\varepsilon^{4}\right), \\
\partial_{t} e+\operatorname{div}((P+e I) u)+\operatorname{div} S-\left(\frac{d}{2}+1\right) \operatorname{div} U-n u \cdot \nabla V & =O\left(\varepsilon^{4}\right),
\end{aligned}
$$

where $I$ is the identity matrix, $u=(n u) / n$, and

$$
P=T\langle s \otimes s\rangle, \quad S=\frac{1}{2} T^{3 / 2}\left\langle|s|^{2} s\right\rangle, \quad U=T^{3 / 2}\langle s\rangle .
$$

Proof. Using the expansion (2.1), elementary integrations yield for $i, j=1, \ldots, d$,

$$
\begin{aligned}
\langle 1\rangle & =T^{d / 2} e^{A} \int_{\mathbb{R}^{d}} e^{-|s|^{2} / 2} d s+O\left(\varepsilon^{2}\right)=(2 \pi T)^{d / 2} e^{A}+O\left(\varepsilon^{2}\right), \\
\left\langle s_{i}\right\rangle & =T^{d / 2} e^{A} \int_{\mathbb{R}^{d}} e^{-|s|^{2} / 2} s_{i} d s+O\left(\varepsilon^{2}\right)=O\left(\varepsilon^{2}\right), \\
\left\langle s_{i} s_{j}\right\rangle & =T^{d / 2} e^{A} \int_{\mathbb{R}^{d}} e^{-|s|^{2} / 2} s_{i} s_{j} d s+O\left(\varepsilon^{2}\right)=n \delta_{i j}+O\left(\varepsilon^{2}\right) .
\end{aligned}
$$


The relations $n=\langle 1\rangle,\langle w\rangle=w\langle 1\rangle=n w$, and $n u=\langle p\rangle=\left\langle T^{1 / 2} s+w\right\rangle=T^{1 / 2}\langle s\rangle+n w$ give for the second moments

$$
\begin{aligned}
\langle p \otimes p\rangle & =T\langle s \otimes s\rangle+\left\langle\left(T^{1 / 2} s+w\right) \otimes\left(T^{1 / 2} s+w\right)-\left(T^{1 / 2} s\right) \otimes\left(T^{1 / 2} s\right)\right\rangle \\
& =P+T^{1 / 2}\langle s\rangle \otimes w+T^{1 / 2} w \otimes\langle s\rangle+w \otimes w\langle 1\rangle \\
& =P+\frac{1}{n}\left\langle T^{1 / 2} s+w\right\rangle \otimes\left\langle T^{1 / 2} s+w\right\rangle-\frac{T}{n}\langle s\rangle \otimes\langle s\rangle \\
& =P+n u \otimes u+O\left(\varepsilon^{4}\right),
\end{aligned}
$$

where in the last equality we have employed (3.11). In a similar way, we compute the third moment:

$$
\begin{aligned}
\frac{1}{2}\left\langle|p|^{2} p\right\rangle & =\frac{1}{2} T^{1 / 2}\left\langle\left|T^{1 / 2} s+w\right|^{2} s\right\rangle+\frac{1}{2} w\left\langle|p|^{2}\right\rangle \\
& =\frac{1}{2} T^{3 / 2}\left\langle|s|^{2} s\right\rangle+T\langle s \otimes s\rangle w+\frac{1}{2} T^{1 / 2}|w|^{2}\langle s\rangle+e w \\
& =S+(P+e I) w+\frac{1}{2} T^{1 / 2}|w|^{2}\langle s\rangle .
\end{aligned}
$$

By (3.10) and (3.11), the energy density can be expanded as

$$
e=\frac{1}{2}\left\langle|p|^{2}\right\rangle=\frac{T}{2}\left\langle|s|^{2}\right\rangle+T^{1 / 2} w \cdot\langle s\rangle+\frac{1}{2}|w|^{2}\langle 1\rangle=\frac{d}{2} n T+\frac{1}{2} n|w|^{2}+O\left(\varepsilon^{2}\right) .
$$

Thus, since $w=u-T^{1 / 2}\langle s\rangle / n$ and $P=\frac{d}{2} n T+O\left(\varepsilon^{2}\right)$, we obtain

$$
\begin{aligned}
\frac{1}{2}\left\langle|p|^{2} p\right\rangle & =S+(P+e I) u-\frac{T^{1 / 2}}{n}\left(P+e I-\frac{1}{2} n|w|^{2} I\right)\langle s\rangle \\
& =S+(P+e I) u-\frac{T^{3 / 2}}{n}\left(\left(\frac{d}{2}+1\right) n I+O\left(\varepsilon^{2}\right)\right)\langle s\rangle \\
& =S+(P+e I) u-\left(\frac{d}{2}+1\right) U+O\left(\varepsilon^{4}\right) .
\end{aligned}
$$

This proves the formal equivalence of (3.3)-(3.5) and (3.6)-(3.8).

3.2. Expansion of the quantum exponential. We wish to give asymptotic expansions of $P, S$, and $U$ up to order $O\left(\varepsilon^{4}\right)$. For this, we first need to expand the quantum Maxwellian. This is done by means of the following lemma, which is adopted from $[8]$.

Lemma 3.2. Let $f(x, p)$ be a smooth symbol. Then the quantum exponential $\operatorname{Exp} f$ can be expanded as follows:

$$
\operatorname{Exp} f=e^{f}-\frac{\varepsilon^{2}}{8} e^{f} \mathcal{Q}+O\left(\varepsilon^{4}\right),
$$

where, using Einstein's summation convention,

$$
\begin{aligned}
\mathcal{Q}= & \partial_{x_{i} x_{j}}^{2} f \partial_{p_{i} p_{j}}^{2} f-\partial_{x_{i} p_{j}}^{2} f \partial_{p_{i} x_{j}}^{2} f+\frac{1}{3} \partial_{x_{i} x_{j}}^{2} f \partial_{p_{i}} f \partial_{p_{j}} f \\
& -\frac{2}{3} \partial_{x_{i} p_{j}}^{2} f \partial_{p_{i}} f \partial_{x_{j}} f+\frac{1}{3} \partial_{p_{i} p_{j}}^{2} f \partial_{x_{i}} f \partial_{x_{j}} f .
\end{aligned}
$$


In the situation at hand, the symbol is $f(x, p)=A(x)-|p-w(x)|^{2} / 2 T(x)$. Then we obtain the following result.

Lemma 3.3. The quantum correction (3.13) can be written for $f(x, p)=A(x)-$ $|p-w(x)|^{2} / 2 T(x)$ as follows:

$$
\begin{aligned}
\mathcal{Q}(s)= & T^{-1}\left(X^{0}+X_{i}^{1} s_{i}+X_{i j}^{2} s_{i} s_{j}+X_{i j k}^{3} s_{i} s_{j} s_{k}\right. \\
& \left.+Y^{0}|s|^{2}+Y_{i}^{1}|s|^{2} s_{i}+Y_{i j}^{2}|s|^{2} s_{i} s_{j}+Z^{0}|s|^{4}\right),
\end{aligned}
$$

where the coefficients $X^{i}, Y^{i}$, and $Z$ are defined by

$$
\begin{aligned}
X^{0} & =-\Delta A-\frac{1}{3}|\nabla A|^{2}+\frac{1}{2 T} \operatorname{tr}\left(\widetilde{R}^{\top} \widetilde{R}\right) \\
X_{i}^{1} & =\frac{2}{T^{1 / 2}} \partial_{x_{m}}\left(\frac{1}{3} A-\log T\right) \widetilde{R}_{m i}-\frac{1}{\sqrt{T}} \Delta w_{i}, \\
X_{i j}^{2} & =\frac{1}{3} \partial_{x_{i} x_{j}}^{2} A+\frac{2}{3} \partial_{x_{i}}(\log T) \partial_{x_{j}} A-\partial_{x_{i}}(\log T) \partial_{x_{j}}(\log T)-\frac{1}{3 T}\left(\widetilde{R}^{\top} \widetilde{R}\right)_{i j} \\
X_{i j k}^{3} & =\frac{1}{3 T^{1 / 2}} \partial_{x_{i} x_{j}}^{2} w_{k}, \\
Y^{0} & =\nabla\left(\frac{1}{2} \log T-\frac{1}{3} A\right) \cdot \nabla(\log T)-\frac{1}{2} \Delta(\log T), \\
Y_{i}^{1} & =\frac{1}{3 T^{1 / 2}} \partial_{x_{m}}(\log T) \widetilde{R}_{m i}, \\
Y_{i j}^{2} & =\frac{1}{6}\left(\partial_{x_{i} x_{j}}^{2}(\log T)+\partial_{x_{i}}(\log T) \partial_{x_{j}}(\log T)\right), \\
Z^{0} & =-\frac{1}{12}|\nabla(\log T)|^{2},
\end{aligned}
$$

and $\widetilde{R}_{i j}=\partial_{x_{j}} w_{i}-\partial_{x_{i}} w_{j}$. The symbol "tr" denotes the trace of a matrix.

Proof. The proof consists in computing the relevant derivatives of $f$ with respect to $x_{i}$ and $p_{j}$, namely

$$
\begin{aligned}
\partial_{x_{i}} f= & \partial_{x_{i}} A+T^{-1} \partial_{x_{i}} w_{k}(p-w)_{k}+\frac{1}{2} T^{-2} \partial_{x_{i}} T|p-w|^{2} \\
= & \partial_{x_{i}} A+T^{-1 / 2} \partial_{x_{i}} w_{k} s_{k}+\frac{1}{2} T^{-1} \partial_{x_{i}} T|s|^{2}, \\
\partial_{x_{i} x_{j}}^{2} f= & \partial_{x_{i} x_{j}}^{2} A-T^{-1} \partial_{x_{i}} w_{k} \partial_{x_{j}} w_{k}-T^{-2} \partial_{x_{j}} T \partial_{x_{i}} w_{k}(p-w)_{k}+T^{-1} \partial_{x_{i} x_{j}}^{2} w_{k}(p-w)_{k} \\
& -T^{-2} \partial_{x_{i}} T \partial_{x_{j}} w_{k}(p-w)_{k}-T^{-3} \partial_{x_{i}} T \partial_{x_{j}} T|p-w|^{2}+\frac{1}{2} T^{-2} \partial_{x_{i} x_{j}}^{2} T|p-w|^{2} \\
= & \partial_{x_{i} x_{j}}^{2} A-T^{-1} \partial_{x_{i}} w_{k} \partial_{x_{j}} w_{k}-T^{-3 / 2} \partial_{x_{j}} T \partial_{x_{i}} w_{k} s_{k}+T^{-1 / 2} \partial_{x_{i} x_{j}}^{2} w_{k} s_{k} \\
& -T^{-3 / 2} \partial_{x_{i}} T \partial_{x_{j}} w_{k} s_{k}-T^{-2} \partial_{x_{i}} T \partial_{x_{j}} T|s|^{2}+\frac{1}{2} T^{-1} \partial_{x_{i} x_{j}}^{2} T|s|^{2}, \\
\partial_{p_{i}} f= & -T^{-1}(p-w)_{i}=-T^{-1 / 2} s_{i}, \\
\partial_{p_{i} x_{j}}^{2} f= & T^{-1} \partial_{x_{j}} w_{i}+T^{-2} \partial_{x_{j}} T(p-w)_{i}=T^{-1} \partial_{x_{j}} w_{i}+T^{-3 / 2} \partial_{x_{j}} T s_{i}, \\
\partial_{p_{i} p_{j}}^{2} f= & -T^{-1} \delta_{i j},
\end{aligned}
$$

and the products appearing in the sum (3.13), which are

$$
\begin{aligned}
\partial_{x_{i} x_{j}}^{2} f \partial_{p_{i} p_{j}}^{2} F= & \left(-T^{-1} \Delta A-T^{-3 / 2} \Delta w_{k}+T^{-2}\|\nabla w\|^{2}+2 T^{-5 / 2} \nabla T \cdot \nabla w_{k}\right) s_{k} \\
& +\left(\frac{1}{2} T^{-2} \Delta T-T^{-3}|\nabla T|^{2}\right)|s|^{2}
\end{aligned}
$$




$$
\begin{aligned}
\partial_{x_{i} p_{j}}^{2} f \partial_{p_{i} x_{j}}^{2} f= & T^{-2} \partial_{x_{i}} w_{j} \partial_{x_{j}} w_{i}+2 T^{-5 / 2} \partial_{x_{j}} T \partial_{x_{i}} w_{j} s_{i}+T^{-3} \partial_{x_{i}} T \partial_{x_{j}} T s_{i} s_{j}, \\
\partial_{x_{i} x_{j}}^{2} f \partial_{p_{i}} f \partial_{p_{j}} f= & \left(T^{-1} \partial_{x_{i} x_{j}}^{2} A-T^{-2} \partial_{x_{i}} w_{\ell} \partial_{x_{j}} w_{\ell}\right) s_{i} s_{j} \\
& +\left(T^{-3 / 2} \partial_{x_{i} x_{j}}^{2} w_{k}-2 T^{-5 / 2} \partial_{x_{i}} T \partial_{x_{j}} w_{k}\right) s_{i} s_{j} s_{k} \\
& +\left(\frac{1}{2} T^{-2} \partial_{x_{i} x_{j}}^{2} T-T^{-3} \partial_{x_{i}} T \partial_{x_{j}} T\right)|s|^{2} s_{i} s_{j}, \\
\partial_{x_{i} p_{j}}^{2} f \partial_{p_{i}} f \partial_{x_{j}} f= & -T^{-3 / 2} \partial_{x_{\ell}} A \partial_{x_{i}} w_{\ell} s_{i}-T^{-2} \partial_{x_{i}} T \partial_{x_{j}} A s_{i} s_{j}-T^{-2} \partial_{x_{i}} w_{j} \partial_{x_{j}} w_{k} s_{i} s_{k} \\
& -T^{-5 / 2} \partial_{x_{i}} T \partial_{x_{j}} w_{k} s_{i} s_{j} s_{k}-\frac{1}{2} T^{-5 / 2} \partial_{x_{i}} w_{j} \partial_{x_{j}} T|s|^{2} s_{i} \\
& -\frac{1}{2} T^{-3} \partial_{x_{i}} T \partial_{x_{j}} T|s|^{2} s_{i} s_{j}, \\
\partial_{p_{i} p_{j}}^{2} f \partial_{x_{i}} f \partial_{x_{j}} f= & -T^{-1}|\nabla A|^{2}-2 T^{-3 / 2} \nabla A \cdot \nabla w_{k} s_{k}-T^{-2} \nabla A \cdot \nabla T|s|^{2} \\
& -T^{-2} \nabla w_{k} \cdot \nabla w_{\ell} s_{k} s_{\ell}-T^{-5 / 2} \nabla T \cdot \nabla w_{k}|s|^{2} s_{k}-\frac{1}{4} T^{-3}|\nabla T|^{2}|s|^{4} .
\end{aligned}
$$

Inserting these expressions into (3.13) and simplifying, we arrive at (3.14). $\square$

3.3. Expansion of the moments. The aim of this subsection is to specify the integrals $\left\langle s^{\alpha}\right\rangle$ in order to expand the moments $n, n u$, and $e$. By Lemma 3.2, we obtain

$$
\begin{aligned}
\left\langle s^{\alpha}\right\rangle & =T^{d / 2} \int_{\mathbb{R}^{d}} g(x, s) s^{\alpha} d s \\
& =T^{d / 2} \int_{\mathbb{R}^{d}} e^{A-|s|^{2} / 2}\left(1-\frac{\varepsilon^{2}}{8} \mathcal{Q}(s)\right) s^{\alpha} d s+O\left(\varepsilon^{4}\right) \\
& =(2 \pi T)^{d / 2} e^{A}\left(\left[s^{\alpha}\right]-\frac{\varepsilon^{2}}{8}\left[\mathcal{Q}(s) s^{\alpha}\right]\right)+O\left(\varepsilon^{4}\right),
\end{aligned}
$$

where $[g]$ denotes the integral of a function $g=g(s)$ against the classical Gaussian kernel,

$$
[g]=(2 \pi)^{-d / 2} \int_{\mathbb{R}^{d}} e^{-|s|^{2} / 2} g(s) d s .
$$

Notice that from the expansion

$$
n=\langle 1\rangle=(2 \pi T)^{d / 2} e^{A}\left(1-\frac{\varepsilon^{2}}{8}[\mathcal{Q}(s)]\right)+O\left(\varepsilon^{4}\right)
$$

it follows that

$$
\left\langle s^{\alpha}\right\rangle=n\left(\left[s^{\alpha}\right]+\frac{\varepsilon^{2}}{8}\left([\mathcal{Q}(s)]\left[s^{\alpha}\right]-\left[\mathcal{Q}(s) s^{\alpha}\right]\right)\right)+O\left(\varepsilon^{4}\right) .
$$

Thus it remains to calculate the integrals $\left[\mathcal{Q}(s) s^{\alpha}\right]$.

Integrals of type $\left[s^{\alpha}\right]$ can be computed explicitly. Using

$$
\int_{\mathbb{R}} t^{m} e^{-t^{2} / 2} d t=\sqrt{2 \pi} \times\left\{\begin{aligned}
0 & \text { if } m \text { is odd } \\
1 & \text { if } m=0 \text { or } m=2 \\
3 & \text { if } m=4 \\
15 & \text { if } m=6
\end{aligned}\right.
$$


it becomes a matter of combinatorics to conclude for $i, j, m, n=1, \ldots, d$,

$$
\begin{aligned}
{\left[s_{i} s_{j}\right] } & =\delta_{i j}, \\
{\left[|s|^{2}\right] } & =d \\
{\left[s_{i} s_{j} s_{m} s_{n}\right] } & =\delta_{i j} \delta_{m n}+\delta_{i m} \delta_{j n}+\delta_{i n} \delta_{j m}, \\
{\left[s_{i} s_{j}|s|^{2}\right] } & =(d+2) \delta_{i j}, \\
{\left[|s|^{4}\right] } & =d(d+2), \\
{\left[s_{i} s_{j} s_{m} s_{n}|s|^{2}\right] } & =(d+4)\left(\delta_{i j} \delta_{m n}+\delta_{i m} \delta_{j n}+\delta_{i n} \delta_{j m}\right), \\
{\left[s_{m} s_{n}|s|^{4}\right] } & =(d+2)(d+4) \delta_{m n} .
\end{aligned}
$$

Then the expansion of $\mathcal{Q}(s)$, given in (3.14), yields the following formulas:

$$
\begin{aligned}
{[\mathcal{Q}(s)] } & =X^{0}+\sum_{\ell} X_{\ell \ell}^{2}+d Y^{0}+(d+2) \sum_{\ell} Y_{\ell \ell}^{2}+d(d+2) Z^{0} \\
{\left[\mathcal{Q}(s) s_{m}\right] } & =X_{m}^{1}+\sum_{\ell}\left(X_{m \ell \ell}^{3}+X_{\ell m \ell}^{3}+X_{\ell \ell m}^{3}\right)+(d+2) Y_{m}^{1} \\
{\left[\mathcal{Q}(s) s_{m}^{2}\right] } & =[\mathcal{Q}(s)]+2 X_{m m}^{2}+2 Y^{0}+2 \sum_{\ell} Y_{\ell \ell}^{2}+2(d+4) Y_{m m}^{2}+4(d+2) Z^{0},(3.19) \\
{\left[\mathcal{Q}(s) s_{m} s_{n}\right] } & =\left(X_{m n}^{2}+X_{n m}^{2}\right)+(d+4)\left(Y_{m n}^{2}+Y_{n m}^{2}\right) \\
{\left[\mathcal{Q}(s)|s|^{2} s_{m}\right] } & =(d+2) X_{m}^{1}+(d+4) \sum_{\ell}\left(X_{m \ell \ell}^{3}+X_{\ell m \ell}^{3}+X_{\ell \ell m}^{3}\right)+(d+2)(d+4) Y_{m}^{1} .
\end{aligned}
$$

LEMma 3.4. The moments $n$, nu, and e can be expressed in terms of the Lagrange multipliers $A, w$, and $T$ asymptotically as follows:

$$
\begin{aligned}
n= & (2 \pi T)^{d / 2} e^{A}-\frac{\varepsilon^{2}}{24 T}(2 \pi T)^{d / 2} e^{A}\left\{-2 \Delta A-|\nabla A|^{2}+(d-2) \nabla \log T \cdot \nabla A(3)\right. \\
& \left.\quad-(d-1) \Delta \log T-\left(\frac{d}{2}-1\right)\left(\frac{d}{2}-2\right)|\nabla \log T|^{2}+\frac{1}{2 T} \operatorname{tr}\left(\widetilde{R}^{\top} \widetilde{R}\right)\right\}+O\left(\varepsilon^{4}\right), \\
n u= & n w+T^{-1} U, \\
e= & \frac{d}{2} n T+\frac{1}{2} n|u|^{2}-\frac{\varepsilon^{2}}{24} n\left\{\Delta \log n-\frac{1}{T} \operatorname{tr}\left(\widetilde{R}^{\top} \widetilde{R}\right)+\frac{d}{2}|\nabla \log T|^{2}\right. \\
& \quad-\Delta \log T-\nabla \log T \cdot \nabla \log n\}+O\left(\varepsilon^{4}\right) .
\end{aligned}
$$

Notice that (3.22) and (3.23) imply the inverse relations

$$
A=\log n-\frac{d}{2} \log T-\frac{d}{2} \log (2 \pi)+O\left(\varepsilon^{2}\right), \quad w=u+O\left(\varepsilon^{2}\right) .
$$

In particular, the vorticity matrices $\widetilde{R}$ and $R$, defined in (1.6), coincide up to order $O\left(\varepsilon^{2}\right)$ since $\widetilde{R}_{i j}=\partial_{j} u_{i}-\partial_{i} u_{j}+O\left(\varepsilon^{2}\right)=R_{i j}+O\left(\varepsilon^{2}\right)$.

Proof. The formula for the particle density (3.22) is obtained by first substituting the expressions for the coefficients $X, Y$, and $Z$ into $(3.17)$. This yields $[\mathcal{Q}(s)]$ in terms of $A, w$, and $T$. Inserting the result into (3.15) then gives (3.22).

In order to derive (3.23), we write, by the definition of $U$ (see (3.9)),

$$
n u=\left\langle T^{1 / 2} s+w\right\rangle=T^{1 / 2}\langle s\rangle+w\langle 1\rangle=T^{-1} U+n w .
$$


Hence, $u-w=U / n T=O\left(\varepsilon^{2}\right)$. The above equations also show that $T^{1 / 2} w \cdot\langle s\rangle=$ $n u \cdot w-n|w|^{2}$. Hence, using $\langle 1\rangle=n$,

$$
\begin{aligned}
e & =\frac{1}{2}\left\langle\left|T^{1 / 2} s+w\right|^{2}\right\rangle=\frac{T}{2}\left\langle|s|^{2}\right\rangle+T^{1 / 2} w \cdot\langle s\rangle+\frac{1}{2}|w|^{2}\langle 1\rangle \\
& =\frac{T}{2}\left\langle|s|^{2}\right\rangle+n u \cdot w-\frac{1}{2} n|w|^{2}=\frac{T}{2}\left\langle|s|^{2}\right\rangle+\frac{1}{2} n|u|^{2}-\frac{1}{2} n|u-w|^{2} .
\end{aligned}
$$

In view of (3.25), we have $|u-w|^{2}=O\left(\varepsilon^{4}\right)$ from which we conclude

$$
e=\frac{T}{2}\left\langle|s|^{2}\right\rangle+\frac{1}{2} n|u|^{2}+O\left(\varepsilon^{4}\right) .
$$

The bracket $\left\langle|s|^{2}\right\rangle$ can be computed from (3.16), employing $\left[|s|^{2}\right]=d$,

$$
\left\langle|s|^{2}\right\rangle=d n+\frac{\varepsilon^{2}}{8} n \sum_{m}\left([\mathcal{Q}(s)]-\left[\mathcal{Q}(s) s_{m}^{2}\right]\right)+O\left(\varepsilon^{4}\right) .
$$

Substitution of (3.17) and (3.19) into the above expression and elimination of $A$ and $w$ using (3.25), gives $\left\langle|s|^{2}\right\rangle$ in terms of $n, n u$, and $T$. This finally leads to (3.24).

3.4. Expansion of the terms $P, S$, and $U$. The QHD equations (3.6)-(3.8) are determined by the following expansion of the auxiliary terms $P, S$, and $U$, defined in (3.9), in terms of the macroscopic variables $n, n u$, and $e$.

LEммa 3.5. The following expansion holds:

$$
\begin{aligned}
P= & n T I+\frac{\varepsilon^{2}}{12} n\left\{\left(\frac{d}{2}+1\right) \nabla \log T \otimes \nabla \log T-\nabla \log T \otimes \nabla \log n\right. \\
& \left.-\nabla \log n \otimes \nabla \log T-(\nabla \otimes \nabla) \log \left(n T^{2}\right)+\frac{R^{\top} R}{T}\right\} \\
& +\frac{\varepsilon^{2}}{12} T \operatorname{div}\left(n \frac{\nabla \log T}{T}\right) I+O\left(\varepsilon^{4}\right), \\
S= & -\frac{\varepsilon^{2}}{12} n\left\{\left(\frac{d}{2}+1\right) R \nabla \log \left(\frac{n}{T}\right)+\left(\frac{d}{2}+2\right) \operatorname{div} R+\frac{3}{2} \Delta u\right\}+O\left(\varepsilon^{4}\right), \\
U= & -\frac{\varepsilon^{2}}{12} n\left\{R \nabla \log \left(\frac{n}{T^{2}}\right)+\operatorname{div} R\right\}+O\left(\varepsilon^{4}\right) .
\end{aligned}
$$

Proof. We apply formula (3.16) to obtain for all $m, n=1, \ldots, d$,

$$
\begin{aligned}
P_{m n} & =n T\left(\delta_{m n}+\frac{\varepsilon^{2}}{8}\left(\delta_{m n}[\mathcal{Q}(s)]-\left[\mathcal{Q}(s) s_{m} s_{n}\right]\right)\right), \\
S_{m} & =-\frac{\varepsilon^{2}}{16} n T^{3 / 2}\left[\mathcal{Q}(s)|s|^{2} s_{m}\right], \\
U_{m} & =-\frac{\varepsilon^{2}}{8} n T^{3 / 2}\left[\mathcal{Q}(s) s_{m}\right] .
\end{aligned}
$$

Then the components of $P$ are computed by employing (3.17) and (3.20), substituting the definitions of the coefficients $X, Y$, and $Z$, and replacing $A$ and $w$ by $n$ and $n u$ according to (3.25). In a similar way, $S$ and $U$ are evaluated using (3.18) and (3.21). 
3.5. Discussion of the QHD equations. The differences between our QHD equations and Gardner's model can be understood as follows. In both approaches, closure is obtained by assuming that the Wigner function $f$ is in thermal equilibrium. However, the notion of "thermal equilibrium" is different.

In order to illustrate the differences, we recall the classical situation. For a system with the Hamiltonian $h(x, p)=|p|^{2} / 2+V(x)$, the unconstrained thermal equilibrium distribution is given by the Gibbs measure $f_{G}(x, p)=\exp \left(-h(x, p) / T_{0}\right)$ which minimizes the relative entropy $S=\int f\left(\log f-1-h / T_{0}\right) d p$. Here, $T_{0}$ denotes a temperature constant. If mass, momentum, and energy densities are given, the constrained thermal equilibrium is realized by a suitable rescaling and a momentum-shift of the Gibbs state,

$$
\widetilde{f}_{G}(x, p)=n(x) \exp \left(-\frac{h(x, p-u(x))}{T(x)}\right) .
$$

The temperature $T(x)$, which is a Lagrange multiplier coming from the minimization procedure, is determined from the given energy density. The choice of $\widetilde{f}_{G}$ as a thermal equilibrium function has its physical justification in the fact that it is the unique minimizer of the relative entropy $S$ with the prescribed moments.

Analogously, a quantum system, which is characterized by its energy operator $H=W^{-1}(h)$ (recall that $W^{-1}$ is the Weyl quantization), attains its minimum of the relative (von Neumann) entropy in the mixed state with Wigner function $f_{Q}=$ $\operatorname{Exp}\left(-h / T_{0}\right)$. This state represents the unconstrained quantum thermal equilibrium. The expansion of $f_{Q}$ in terms of the scaled Planck constant $\varepsilon^{2}$ was first given in [30],

$$
f_{Q}(x, p)=\exp \left(-h(x, p) / T_{0}\right)\left(1+\varepsilon^{2} f_{2}(x, p)\right)+O\left(\varepsilon^{4}\right)
$$

with an appropriate function $f_{2}$. As a definition of the quantum equilibrium with moment constraints, Gardner employed this expansion of $f_{Q}$ and modified it as follows:

$$
\widetilde{f}_{Q}(x, p)=n(x) \exp \left(-\frac{h(x, p-u(x))}{T(x)}\right)\left(1+\varepsilon^{2} f_{2}(x, p-u(x))\right)+O\left(\varepsilon^{4}\right) .
$$

These modifications mimic the passage from the Gibbs state to (3.29) in the classical situation. The use of $\widetilde{f}_{Q}$ as an equilibrium function results in simple formulas for the moment equations. However, the Wigner function (3.30) is an ad hoc ansatz. Moreover, in contrast to the classical case, $\widetilde{f}_{Q}$ is not the constrained minimizer for the relative von Neumann entropy.

The equilibrium state $M_{f}$ used here is a genuine minimizer of the relative quantum entropy with respect to the given moments. In the spirit of the classical situation, these equilibria seem to be more natural. The price we have to pay is the appearance of various additional terms in the expansion of $M_{f}$.

If the temperature is assumed to be constant and if only the particle density is prescribed, both approaches to define a thermal equilibrium coincide. In order to see this, we write Gardner's momentum-shifted quantum equilibrium more explicitly than in (3.30):

$\widetilde{f}_{G}(x, p, t)=e^{-V / T-|p|^{2} / 2 T}\left\{1+\frac{\varepsilon^{2}}{8 T^{2}}\left(-\Delta V+\frac{1}{3 T}|\nabla V|^{2}+\frac{1}{3 T} p_{i} p_{j} \partial_{x_{i} x_{j}} V\right)\right\}+O\left(\varepsilon^{4}\right)$,

The equilibrium function obtained from the entropy minimization with given particle 
density equals (see [20], Remark 3.3)

$$
\begin{aligned}
\tilde{f}(x, p, t) & =\operatorname{Exp}\left(A(x, t)-\frac{|p|^{2}}{2 T}\right) \\
& =e^{A-|p|^{2} / 2 T}\left\{1+\frac{\varepsilon^{2}}{8 T}\left(\Delta A+\frac{1}{3}|\nabla A|^{2}-\frac{1}{3 T} p_{i} p_{j} \partial_{x_{i} x_{j}} A\right)\right\}+O\left(\varepsilon^{4}\right) .
\end{aligned}
$$

Both approximations are essentially derived in the same way. Using $n=\int \widetilde{f}_{Q} d p=$ $(2 \pi T)^{d / 2} e^{-V / T}+O\left(\varepsilon^{2}\right)$ and assuming constant (or "slowly varying") temperature, Gardner has substituted $\nabla V=-T \nabla \log n+O\left(\varepsilon^{2}\right)$ in the formula for $\widetilde{f}_{Q}$ in order to avoid the second-order derivatives of the potential. This substitution in fact yields the approximation $\widetilde{f}$ since, by $(3.25), \nabla A=\nabla \log n+O\left(\varepsilon^{2}\right)$, and thus, both expansions $\widetilde{f}_{Q}$ and $\tilde{f}$ coincide.

4. Simplified QHD models. The full QHD model is given by equations (3.6)(3.8) with the constitutive relations (3.26)-(3.28). In this section we will discuss some simplified versions. The QHD equations read, up to order $O\left(\varepsilon^{4}\right)$,

$$
\begin{aligned}
\partial_{t} n+\operatorname{div}(n u) & =0, \\
\partial_{t}(n u)+\operatorname{div}(n u \otimes u)+\operatorname{div} P-n \nabla V & =0, \\
\partial_{t} e+\operatorname{div}((P+e I) u)+\operatorname{div} S-\left(\frac{d}{2}+1\right) \operatorname{div} U-n u \cdot \nabla V & =0,
\end{aligned}
$$

where $e$ is the energy density given by (3.24), and $P, S$, and $U$ are given by (3.26)(3.28) (without the $O\left(\varepsilon^{4}\right)$ terms).

First, we shall assume that the temperature is slowly varying in the sense of $\nabla \log T=O\left(\varepsilon^{2}\right)$. Then the expressions $\varepsilon^{2} \nabla \log T$ in (3.26)-(3.28) are of order $O\left(\varepsilon^{4}\right)$ and can therefore be neglected in our approximation:

$$
\begin{aligned}
P & =n T I-\frac{\varepsilon^{2}}{12} n(\nabla \otimes \nabla) \log n, \\
S & =-\frac{\varepsilon^{2}}{12} n\left\{\left(\frac{d}{2}+1\right) R \nabla \log n+\left(\frac{d}{2}+2\right) \operatorname{div} R+\frac{3}{2} \Delta u\right\}, \\
U & =-\frac{\varepsilon^{2}}{12} n\{R \nabla \log n+\operatorname{div} R\}, \\
e & =\frac{d}{2} n T+\frac{1}{2} n|u|^{2}-\frac{\varepsilon^{2}}{24} n\left(\Delta \log n-\frac{1}{T} \operatorname{tr}\left(R^{\top} R\right)\right),
\end{aligned}
$$

As in [11], the stress tensor $P$ consists of the classical pressure $n T$ on the diagonal and the "quantum pressure" $\left(\varepsilon^{2} / 12\right) n(\nabla \otimes \nabla) \log n$. The terms $S$ and $U$ provide additional quantum corrections not present in [11]. The energy density consists of the thermal energy, kinetic energy, and quantum energy. Again, due to the vorticity $R$, the energy takes a different form than the expressions in [11, 15].

Further simplifications can be obtained if the vorticity is "small", i.e. $R=O\left(\varepsilon^{2}\right)$. In one space dimension this term always vanishes. If $R=O\left(\varepsilon^{2}\right)$ then $\varepsilon^{2} R$ is of order $O\left(\varepsilon^{4}\right)$ and can be neglected. We obtain the QHD equations

$$
\begin{array}{r}
\partial_{t} n+\operatorname{div}(n u)=0, \\
\partial_{t}(n u)+\operatorname{div}(n u \otimes u)+\nabla(n T)-\frac{\varepsilon^{2}}{12} \operatorname{div}(n(\nabla \otimes \nabla) \log n)-n \nabla V=0, \\
\partial_{t} e+\operatorname{div}((P+e I) u)-\frac{\varepsilon^{2}}{8} \operatorname{div}(n \Delta u)-n u \cdot \nabla V=0,
\end{array}
$$


with the stress tensor and energy density, respectively,

$$
P=n T I-\frac{\varepsilon^{2}}{12} n(\nabla \otimes \nabla) \log n, \quad e=\frac{d}{2} n T+\frac{1}{2} n|u|^{2}-\frac{\varepsilon^{2}}{24} n \Delta \log n .
$$

This system of equations corresponds to Gardner's QHD model except for the dispersive velocity term $\left(\varepsilon^{2} / 8\right) \operatorname{div}(n \Delta u)$. We already mentioned in the introduction that this term has been also derived by Gardner and Ringhofer [15] by employing a Chapman-Enskog expansion of the Wigner-Boltzmann equation. They do not obtain vorticity terms since they assume that the quantum equilibrium distribution is an even function of the momentum $p$. Roughly speaking, this gives (in our context) the quantum exponential $\operatorname{Exp}\left(A-|p|^{2} / 2 T\right)$ instead of $\operatorname{Exp}\left(A-|p-w|^{2} / 2 T\right)$. The Lagrange multiplier $w$, however, is responsible for the presence of the vorticity term $R$.

Interestingly, most quantum terms cancel out in the energy equation. In fact, by substituting the above expression for the energy density in (4.10), a computation yields

$$
\partial_{t}(n T)+\operatorname{div}(n T u)+\frac{2}{d} n T \operatorname{div} u-\frac{\varepsilon^{2}}{6 d} \operatorname{div}(n \Delta u)=0 .
$$

5. Conserved quantities. In this subsection we show that the mass and energy are conserved for the system (3.6)-(3.8) and (1.2) with the relations (3.26)-(3.28), neglecting the $O\left(\varepsilon^{4}\right)$ terms.

LEMMA 5.1. The mass $N(t)=\int n d x$ and the energy

$$
E(t)=\int_{\mathbb{R}^{d}}\left(e+\frac{\lambda^{2}}{2}|\nabla V|^{2}\right) d x
$$

where e is defined in (3.24) (without the $O\left(\varepsilon^{4}\right)$ term), are conserved, i.e. $d N / d t(t)=0$ and $d E(t) / d t=0$ for all $t>0$. Furthermore, the energy can be written as

$$
\begin{aligned}
E(t)= & \int_{\mathbb{R}^{d}}\left(\frac{d}{2} n T+\frac{1}{2} n|u|^{2}+\frac{\lambda^{2}}{2}|\nabla V|^{2}+\frac{\varepsilon^{2}}{6}|\nabla \sqrt{n}|^{2}+\frac{\varepsilon^{2} d}{48} n|\nabla \log T|^{2}\right. \\
& \left.+\frac{\varepsilon^{2}}{24 T} n \operatorname{tr}\left(R^{\top} R\right)\right) d x \geq 0 .
\end{aligned}
$$

Proof. The conservation of $N$ is clear. In order to prove that $E$ is conserved, we differentiate $E$ and employ the equations (4.3) and (1.2):

$$
\begin{aligned}
\frac{d E}{d t} & =\int_{\mathbb{R}^{d}}\left(\partial_{t} e+\lambda^{2} \nabla V \cdot \nabla \partial_{t} V\right) d x=\int_{\mathbb{R}^{d}}\left(n u \cdot \nabla V-\lambda^{2} V \partial_{t} \Delta V\right) d x \\
& =\int_{\mathbb{R}^{d}}\left(-\operatorname{div}(n u) V-V \partial_{t} n\right) d x=0,
\end{aligned}
$$

taking into account (4.1). Next we show the formulation (5.1). The integral of the energy density $e$ can be written as

$$
\begin{aligned}
E= & \int_{\mathbb{R}^{d}}\left(\frac{d}{2} n T+\frac{1}{2} n|u|^{2}+\frac{\varepsilon^{2} d}{48} n|\nabla \log T|^{2}+\frac{\varepsilon^{2}}{24 T} n \operatorname{tr}\left(R^{\top} R\right)\right) d x \\
& +\frac{\varepsilon^{2}}{24} \int_{\mathbb{R}^{d}}(-n \Delta \log n+n \Delta \log T+n \nabla \log T \cdot \nabla \log n) d x
\end{aligned}
$$


The last integral equals, after an integration by parts, to

$$
\frac{\varepsilon^{2}}{24} \int_{\mathbb{R}^{d}}\left(4|\nabla \sqrt{n}|^{2}-\nabla n \cdot \nabla \log T+n \nabla \log T \cdot \nabla \log n\right) d x=\frac{\varepsilon^{2}}{6} \int_{\mathbb{R}^{d}}|\nabla \sqrt{n}|^{2} d x
$$

which shows (5.1). प

The energy (5.1) consists of, in this order, the thermal energy, the kinetic energy, the electric energy, the quantum energy associated to the Bohm potential, and two additional quantum energy terms associated to spatial variations of the temperature and the vorticity. The last two energy terms are new, i.e., they do not appear in the QHD equations of [11].

In the case of the QHD equations with slowly varying temperature, i.e. equations (4.1)-(4.3) and (1.2) with the definitions (4.4)-(4.6), the energy is given by (5.1) except the term involving $|\nabla \log T|^{2}$. If, additionally, the vorticity is "small", i.e. in the case of the model (4.8)-(4.10) and (1.2), which is used in the numerical simulations of section 6 , the energy is equal to (5.1) except the last two terms.

6. Numerical results. In this section we summarize the results from our numerical simulations of a simple one-dimensional GaAs resonant tunneling diode, using the new QHD system. The aim is to compare the new equations with Gardner's QHD model; in particular, the influence of the dispersive velocity term will be explored.

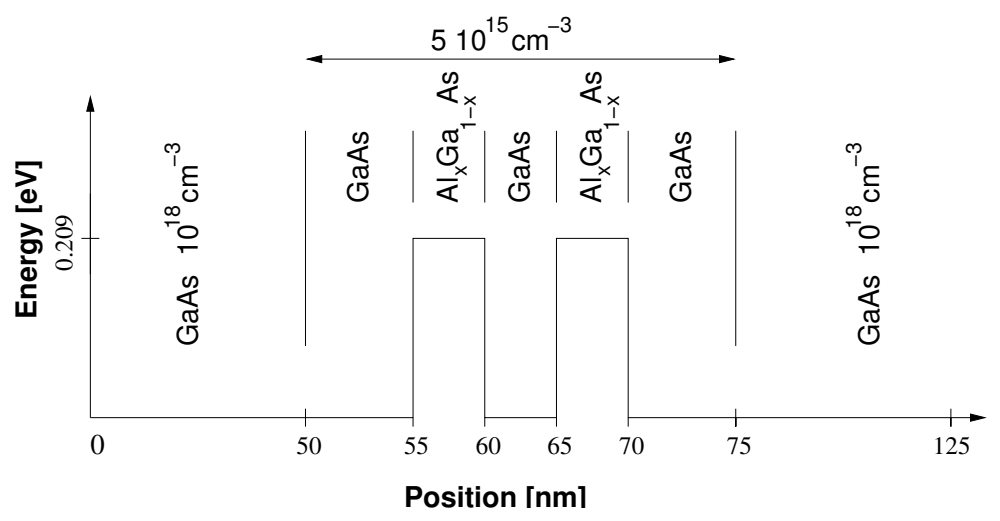

FIG. 6.1. Geometry of the resonant tunneling diode and external potential modeling the double barriers. The Al mole fraction is $x=0.3$.

The geometry of the tunneling diode is chosen essentially as in [11] (see Figure 6.1). The diode consists of highly doped $50 \mathrm{~nm}$ GaAs regions near the contacts and a lightly doped middle region (the channel) of $25 \mathrm{~nm}$ length. The channel contains a quantum well of $5 \mathrm{~nm}$ length sandwiched between two $5 \mathrm{~nm} \mathrm{Al}_{x} \mathrm{Ga}_{1-x} \mathrm{As}$ barriers with Al mole fraction $x=0.3$. The double barrier heterostructure is placed between two $5 \mathrm{~nm}$ GaAs spacer layers. The total length of the device is thus $125 \mathrm{~nm}$. The double barrier height is $B=0.209 \mathrm{eV}$. It is incorporated into the QHD equations by replacing $V$ by $V+B$.

For our simulations, we use the one-dimensional stationary QHD equations for small temperature variations $\nabla \log T=O\left(\varepsilon^{2}\right)$ coupled to the Poisson equation for the 


\begin{tabular}{|l|l|l|}
\hline Parameter & Physical meaning & Value \\
\hline$q$ & elementary charge & $1.602 \cdot 10^{-19} \mathrm{As}$ \\
$m$ & effective electron mass & $0.067 \cdot 10^{-31} \mathrm{~kg}$ \\
$k_{B}$ & Boltzmann constant & $1.3807 \cdot 10^{-23} \mathrm{~kg} \mathrm{~m}^{2} / \mathrm{s}^{2} \mathrm{~K}$ \\
$\hbar$ & reduced Planck constant & $1.0546 \cdot 10^{-34} \mathrm{~kg} \mathrm{~m}^{2} / \mathrm{s}$ \\
$\varepsilon_{s}$ & semiconductor permitivity & $12.9 \cdot 8.8542 \cdot 10^{-12} \mathrm{~A}^{2} \mathrm{~s}^{4} / \mathrm{kg} \mathrm{m}^{3}$ \\
$\tau_{0}$ & momentum relaxation time & $0.9 \cdot 10^{-12} \mathrm{~s}$ \\
$T_{0}$ & lattice temperature & $77 \mathrm{~K}$ \\
\hline \multicolumn{2}{|r}{ TABLe 6.1} \\
\hline
\end{tabular}

electric potential. Including the physical parameters, these equations read as follows:

$$
\begin{aligned}
(n u)_{x} & =0 \\
m\left(n u^{2}\right)_{x}+k_{B}(n T)_{x}-\frac{\hbar^{2}}{12 m}\left(n(\log n)_{x x}\right)_{x}-q n V_{x} & =0 \\
\frac{5}{2} k_{B}(n T u)_{x}+\frac{1}{2} m\left(n u^{3}\right)_{x}-\frac{\hbar^{2}}{8 m}\left(n u(\log n)_{x x}+n u_{x x}\right)_{x}-q n u V_{x} & =k_{B} \sigma\left(n T_{x}\right)_{x} \\
\varepsilon_{s} V_{x x} & =q(n-C)
\end{aligned}
$$

We have allowed the heat flux $k_{B} \sigma\left(n T_{x}\right)_{x}$ since this term has also been used by Gardner [11] in his model with which we wish to compare our numerical results. The physical constants in the above equations are the effective mass $m$, the Boltzmann constant $k_{B}$, the reduced Planck constant $\hbar$, the elementary charge $q$, and the semiconductor permittivity $\varepsilon_{s}$. The values of these constants are given in Table 6.1. The parameter $\sigma$ is defined by

$$
\sigma=\kappa \tau_{0} \frac{k_{B} T_{0}}{m},
$$

with the thermal conductivity $\kappa$, the relaxation time $\tau_{0}$, and the lattice temperature $T_{0}$. Using a standard scaling (see, e.g., [18]), we derive the scaled QHD equations (1.2)-(1.5) of the introduction, where the nondimensional parameters are given by

$$
\varepsilon^{2}=\frac{\hbar^{2}}{m k_{B} T_{0} L^{2}}, \quad \lambda^{2}=\frac{\varepsilon_{s} k_{B} T_{0}}{q^{2} C^{*} L^{2}} .
$$

Here, $L$ is the device length and $C^{*}$ the maximal doping concentration. For the values we used in the numerical simulations below (see Table 6.1 ), we obtain $\varepsilon^{2} \approx 0.011$ which justifies our expansion in $\varepsilon^{2}$.

We compare the numerical results with Gardner's QHD equations which do not contain the dispersive expression (1.7) in the velocity but additional relaxation-time terms of Baccarani-Wordeman type [3]:

$$
\begin{gathered}
(n u)_{x}=0 \\
m\left(n u^{2}\right)_{x}+k_{B}(n T)_{x}-\frac{\hbar^{2}}{12 m}\left(n(\log n)_{x x}\right)_{x}-q n V_{x}=-\frac{m n u}{\tau_{p}} \\
\frac{5}{2} k_{B}(n T u)_{x}+\frac{1}{2} m\left(n u^{3}\right)_{x}-\frac{\hbar^{2}}{8 m}\left(n u(\log n)_{x x}\right)_{x}-q n u V_{x} \\
=k_{B} \sigma\left(n T_{x}\right)_{x}-\frac{1}{\tau_{w}}\left(e-\frac{3}{2} n T_{0}\right)
\end{gathered}
$$


together with the Poisson equation (6.4). Here, the momentum and energy relaxation times are given by, respectively,

$$
\tau_{p}=\tau_{0} \frac{T_{0}}{T}, \quad \tau_{w}=\frac{\tau_{p}}{2}\left(1+\frac{3 T}{m v_{s}^{2}}\right),
$$

where $\tau_{0}$ is given in Table 6.1 and $v_{s}=2 \cdot 10^{7} \mathrm{~cm} / \mathrm{s}$ is the saturation velocity.

The above QHD equations have to be solved in the interval $(0,1)$ with the following boundary conditions taken from [11]:

$$
\begin{gathered}
n(0)=C(0), \quad n(1)=C(1), \quad n_{x}(0)=n_{x}(1)=0, \\
u_{x}(0)=u_{x}(1)=0, \quad T(0)=T(1)=T_{0}, \quad V(0)=0, \quad V(1)=U_{0},
\end{gathered}
$$

where $U_{0}$ is the applied voltage.

First, we discretize the new QHD equations (6.1)-(6.4) using central finite differences on a uniform mesh with $N=500$ points. This corresponds to a mesh size of $\triangle x=1 / 500=0.002$. The resulting discrete nonlinear system is solved by a damped Newton method with damping parameter found by a line search method (see Algorithm A6.3.1 in [9]). We employ the following continuation method for the applied voltage: first the system of equations is solved for applied voltage $U_{0}=0 \mathrm{~V}$; then given the solution corresponding to the voltage $U_{0}$, it is taken as an initial guess for the solution of the system with applied voltage $U_{0}+\triangle U$. The voltage step is chosen as $\triangle U=1 \mathrm{mV}$.

The current-voltage characteristics using the thermal conductivities $\kappa=0.2$ and $\kappa=0.3$ are presented in Figure 6.2. There are two regions of negative differential resistance (NDR) if $\kappa=0.2$ and three NDR regions if $\kappa=0.3$. It is well known for Gardner's QHD model, that the behavior of the solutions is quite sensitive to changes of the value of the thermal conductivity. We observe a similar sensitive dependence: the peak-to-valley ratio, i.e. the ratio of local maximal to local minimal current density, is larger for larger thermal conductivities.

The electron density shows a charge enhancement in the quantum well which is more pronounced for smaller $\kappa$ (see Figure 6.3). At the center of the right barrier, the electron density dramatically decreases. After the first valley in the current-voltage characteristics, the density develops a "wiggle". This phenomenum is not a numerical effect but it is related to the dispersive structure of the equations [26]. For larger values of the thermal conductivity, the minimum of the particle density increases, which stabilizes the numerical scheme.

The influence of the effective mass on the current-voltage curves are shown in Figure 6.4 (left). Corresponding to the effective masses $m=0.067 m_{0}, m=0.092 m_{0}$, $m=0.126 m_{0}$, the peak-to-valley ratios are $1.44,1.79,2.37$, respectively. Similarly to the quantum drift-diffusion model, the peak-to-valley ratio increases with the effective mass [21]. Strictly speaking, the effective mass is not constant in the whole device but it is material depending. The use of a constant effective mass can be justified in an average sense. The correct averaging is not known but the average value could be used as a fitting parameter. The use of a nonconstant effective mass would be more physical, but the modeling and the numerical approximation is - even in the much simpler quantum drift-diffusion model - quite involved [28, 29].

In Figure 6.4 (right) the current-voltage curve for the barrier height $B=0.3 \mathrm{eV}$ is shown. As expected, the peak-to-valley ratio is larger if the barrier is higher (corresponding to a higher $\mathrm{Al}$ mole fraction); the values for the first NDR region are 1.44 


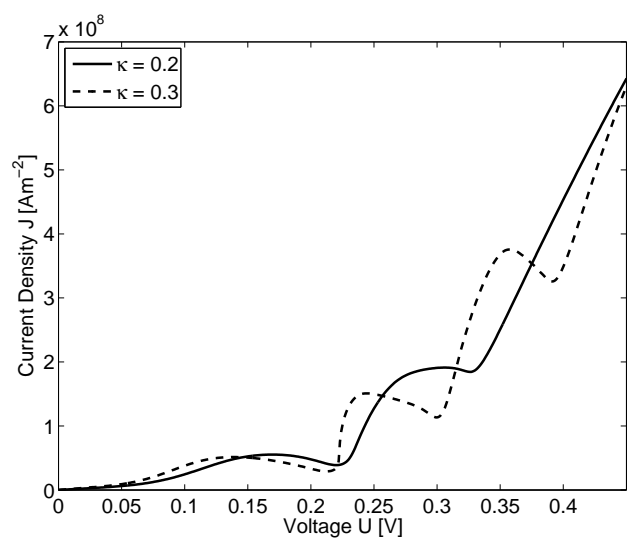

FIG. 6.2. Current-voltage characteristic for the new QHD system with thermal conductivities $\kappa=0.2$ (solid line) and $\kappa=0.3$ (dashed line).
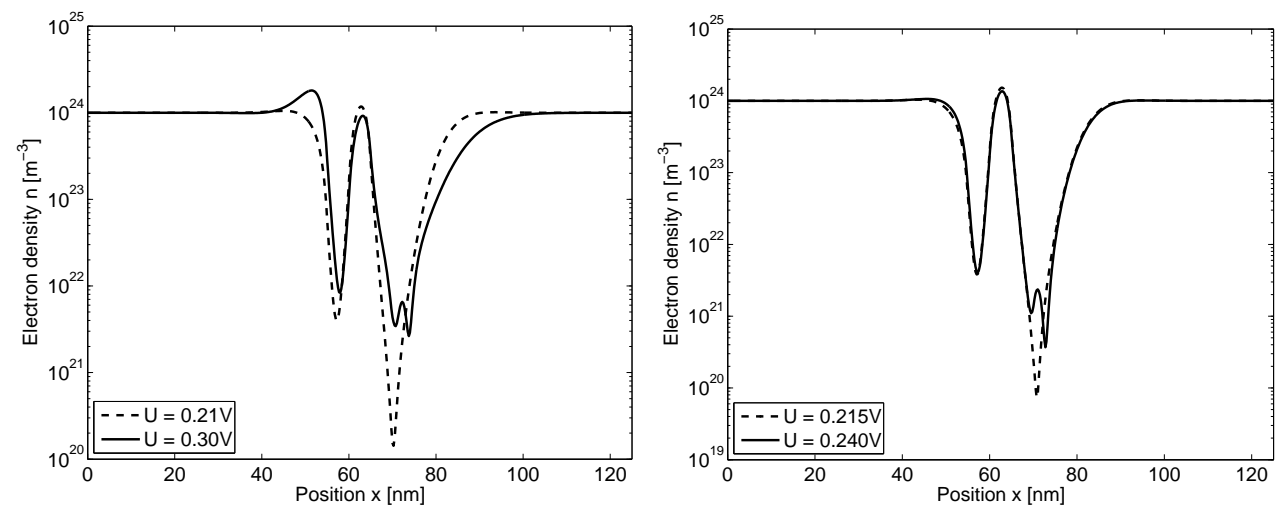

FIG. 6.3. Electron density before (dashed line) and after (solid line) the first valley for thermal conductivities $\kappa=0.2$ (left) and $\kappa=0.3$ (right).

for $B=0.209 \mathrm{eV}$ and 2.48 for $B=0.3 \mathrm{eV}$. The current densities are much smaller than in Figure 6.2, where the lower potential barrier $B=0.209 \mathrm{eV}$ has been used. Interestingly, there are at least three NDR regions, whereas there are only two regions for the barrier height $B=0.209 \mathrm{eV}$.

In Figure 6.5, the current-voltage curves for the new QHD equations and for Gardner's model are compared. Gardner's model is discretized using a second-order upwind method as in [11]. The right figure with $N=500$ points corresponds to Figure 2 of the cited paper. Notice that close to equilibrium, there are well-known difficulties to compute the solution, which is not the case for our new model. Due to the numerical viscosity introduced by the upwind method, it is clear that the solution of Gardner's model depends on the mesh size. The solution to the new QHD equations is less mesh depending. In particular, the numerical results before the first valley are almost the same for $N \geq 500$ grid points. More importantly, the slope of the curve in Gardner's model becomes steeper in the region after the valley when the mesh size $\triangle x$ is decreased. In fact, the numerical scheme becomes unstable for mesh sizes $\triangle x<1 / 750$ which may be explained by the steep slope. On the other hand, the 

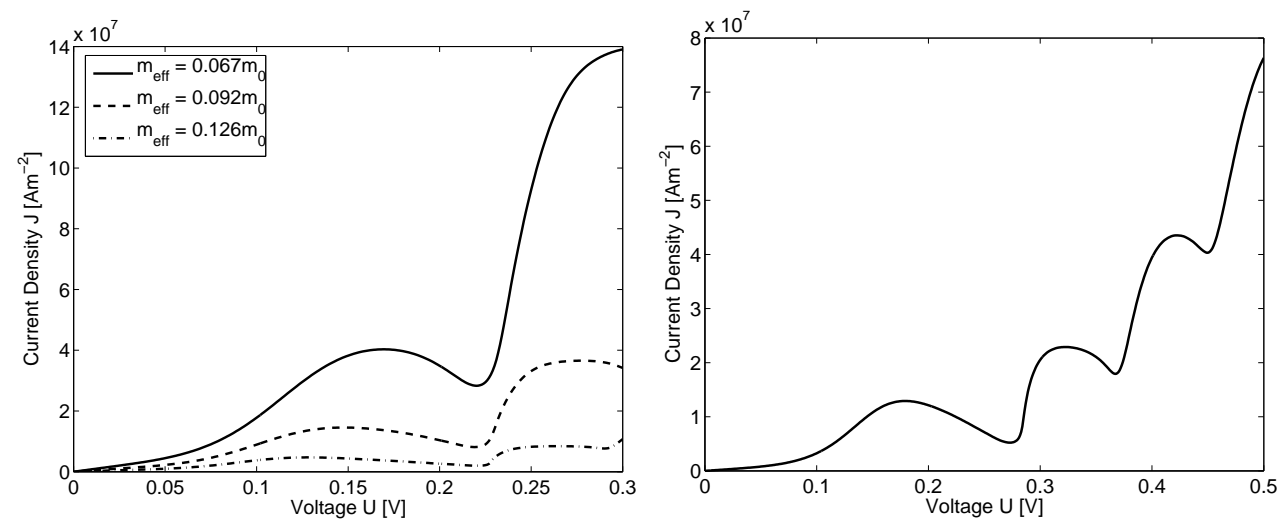

FIG. 6.4. Left: Influence of the effective mass $m_{\text {eff }}$ on the current-voltage characteristic. Right: Current-voltage characteristic for a barrier height of $B=0.3 \mathrm{eV}$. In both pictures, $\kappa=0.2$.

current-voltage curve of the new QHD model does not seem to develop such singular slopes. Moreover, it is possible to solve the discrete system for mesh sizes $\triangle x<1 / 750$ (not shown).

Finally, we study the influence of the dispersive velocity term $\left(\varepsilon^{2} / 8\right)\left(n u_{x x}\right)_{x}$. For this, we replace the factor $\varepsilon^{2} / 8$ by $\delta^{2} / 8$ and choose various values for $\delta$. Clearly, only $\delta=\varepsilon$ corresponds to the physical situation. The dispersive velocity term indeed regularizes the equations in the sense that the current-voltage curves become "smoother" (see Figure 6.6). A similar "smoothing" has been observed in [22, 23] for the viscous QHD equations, but there, the smoothing originates from a diffusive and not from a dispersive term. For smaller values of $\delta$, the peak-to-valley ratio of the first NDR region becomes larger. For $\delta=0$, we arrive at Gardner's QHD equations without relaxation terms. We already mentioned that a central finite-difference discretization fails for this model; therefore, the numerical limit $\delta \rightarrow 0$ cannot be performed.
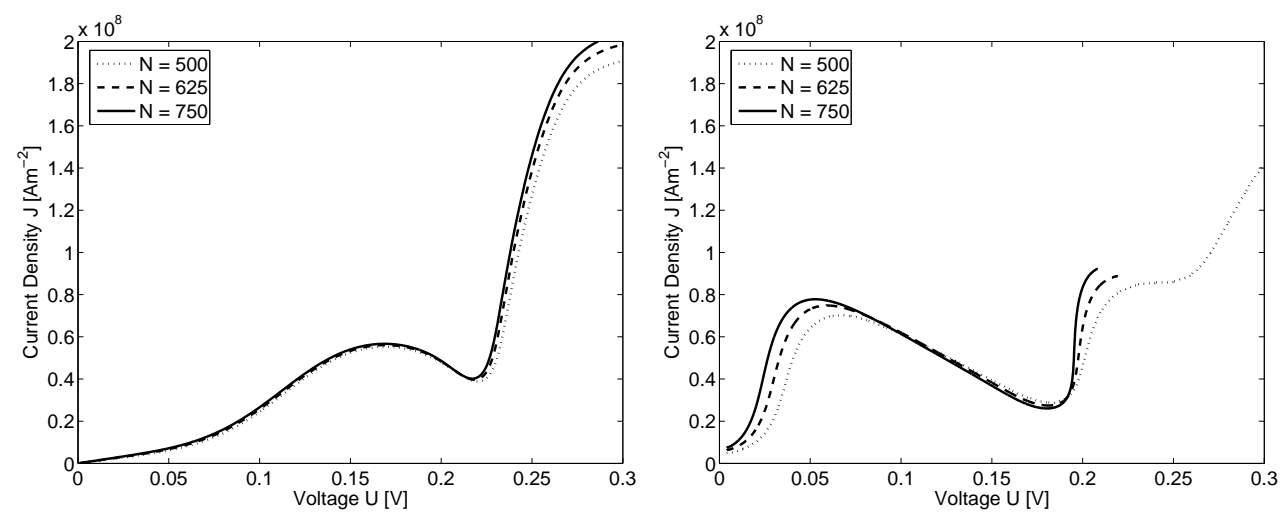

FIG. 6.5. Influence of the number of discretization points on the current-voltage characteristics for the new QHD equations (left) and for Gardner's QHD model (right). In both pictures, $\kappa=0.2$. 


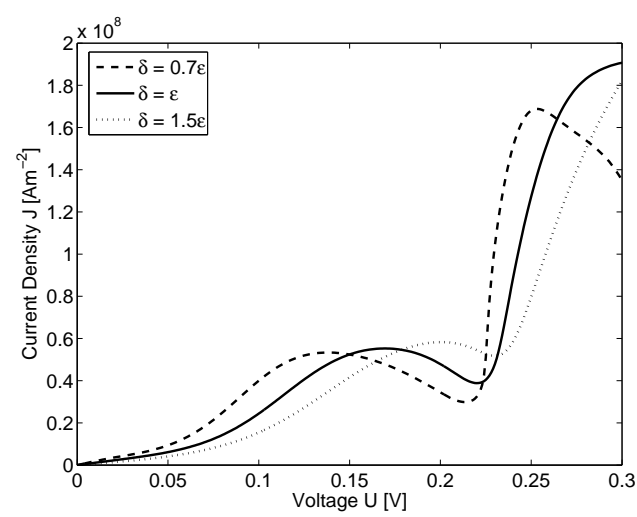

FIG. 6.6. Influence of the dispersive velocity term $\left(\delta^{2} / 8\right)\left(n u_{x x}\right)_{x}$ on the current-voltage curve for thermal conductivity $\kappa=0.2$.

\section{REFERENCES}

[1] M. Ancona and G. Iafrate. Quantum correction to the equation of state of an electron gas in a semiconductor. Phys. Rev. B 39 (1989), 9536-9540.

[2] M. Ancona and H. Tiersten. Macroscopic physics of the silicon inversion layer. Phys. Rev. B 35 (1987), 7959-7965.

[3] G. Baccarani and M. Wordeman. An investigation of steady-state velocity overshoot effects in Si and GaAs devices. Solid-State Electronics 28 (1985), 407-416.

[4] P. Bhatnagar, E. Gross, and M. Krook. A model for collision processes in gases. I. Small amplitude processes in charged and neutral one-component systems. Phys. Review 94 (1954), 511-525.

[5] Z. Chen. A finite element method for the quantum hydrodynamic model for semiconductor devices. Computers Math. Appl. 31 (1996), 17-26.

[6] P. Degond, F. Méhats, and C. Ringhofer. Quantum hydrodynamic models derived from the entropy principle. Contemp. Math. 371 (2005), 107-131.

[7] P. Degond, F. Méhats, and C. Ringhofer. Quantum energy-transport and drift-diffusion models. J. Stat. Phys. 118 (2005), 625-665.

[8] P. Degond and C. Ringhofer. Quantum moment hydrodynamics and the entropy principle. J. Stat. Phys. 112 (2003), 587-628.

[9] J. Dennis and R. Schnabel. Numerical Methods for Unconstrained Optimization and Nonlinear Equations. SIAM, Philadelphia, 1996.

[10] D. Ferry and J.-R. Zhou. Form of the quantum potential for use in hydrodynamic equations for semiconductor device modeling. Phys. Rev. B 48 (1993), 7944-7950.

[11] C. Gardner. The quantum hydrodynamic model for semiconductor devices. SIAM J. Appl. Math. 54 (1994), 409-427.

[12] C. Gardner. Resonant tunneling in the quantum hydrodynamic model. VLSI Design 3 (1995), 201-210.

[13] C. Gardner and C. Ringhofer. Smooth quantum potential for the hydrodynamic model. Phys. Rev. E 53 (1996), 157-167.

[14] C. Gardner and C. Ringhofer. Approximation of thermal equilibrium for quantum gases with discontinuous potentials and application to semiconductor devices. SIAM J. Appl. Math. 58 (1998), 780-805.

[15] C. Gardner and C. Ringhofer. The Chapman-Enskog expansion and the quantum hydrodynamic model for semiconductor devices. VLSI Design 10 (2000), 415-435.

[16] I. Gasser, P. Markowich, D. Schmidt, and A. Unterreiter. Macroscopic theory of charged quantum fluids. In: P. Marcati et al. (eds.). Mathematical Problems in Semiconductor Physics. Research Notes in Mathematics Series 340 (1995), 42-75, Pitman.

[17] H. Grubin and J. Kreskovsky. Quantum moment balance equations and resonant tunneling structures. Solid-State Electronics 32 (1989), 1071-1075.

[18] M. Gualdani and A. Jüngel. Analysis of the viscous quantum hydrodynamic equations for semiconductors. Europ. J. Appl. Math. 15 (2004), 577-595. 
[19] A. Jüngel. Quasi-hydrodynamic Semiconductor Equations. Birkhäuser, Basel, 2001.

[20] A. Jüngel and D. Matthes. A derivation of the isothermal quantum hydrodynamic equations using entropy minimization. Z. Angew. Math. Mech. 85 (2005), 806-814.

[21] A. Jüngel and J.-P. Milišić. Macroscopic quantum models with and without collisions. To appear in Proceedings of the Sixth International Workshop on Mathematical Aspects of Fluid and Plasma Dynamics, Kyoto, Japan. Transp. Theory Stat. Phys., 2005.

[22] A. Jüngel and J.-P. Milišić. Numerical comparison of viscous and non-viscous quantum hydrodynamic models for semiconductors. In preparation, 2005.

[23] A. Jüngel and S. Tang. Numerical approximation of the viscous quantum hydrodynamic model for semiconductors. To appear in Appl. Numer. Math., 2005.

[24] C. Levermore. Moment closure hierarchies for kinetic theories. J. Stat. Phys. 83 (1996), 10211065.

[25] E. Madelung. Quantentheorie in hydrodynamischer Form. Z. Physik 40 (1927), 322-326.

[26] P. Pietra and C. Pohl. Weak limits of the quantum hydrodynamic model. In: Proceedings of the International Workshop on Quantum Kinetic Theory, Breckenridge, Colorado, USA, Special Issue of VLSI Design 9 (1999), 427-434.

[27] C. Ringhofer, C. Gardner, and D. Vasileska. Effective potentials and quantum fluid models: a thermodynamic approach. Intern. J. High Speed Electronics Sys. 13 (2003), 771-801.

[28] A. Wettstein. Quantum Effects in MOS Devices. Series in Microelectronics 94, Hartung-Gorre, Konstanz, 2000.

[29] A. Wettstein, A. Schenk, and W. Fichtner. Quantum device-simulation with the densitygradient model on unstructured grids. IEEE Trans. Electr. Dev. 48 (2001), 279-284.

[30] E. Wigner. On the quantum correction for thermodynamic equilibrium. Phys. Rev. 40 (1932), 749-759. 